\title{
Genetic links to pouch failure
}

The development of Crohn's disease in patients with ulcerative colitis who have undergone ileal pouch-anal anastomosis (IPAA) can have severe consequences. A family history of Crohn's disease is a risk factor for the development of this condition in patients who undergo IPAA, according to a recently published study.

IPAA is the surgical treatment of choice for patients with ulcerative colitis or indeterminate colitis who require colectomy and are not responsive to medication or develop dysplasia. This procedure is not typically performed in patients with known Crohn's disease; however, researchers at the Cleveland Clinic Foundation noted that a considerable proportion of patients developed Crohn's disease or a similar condition after surgery. "A family history of Crohn's disease was associated with an increased risk [of] Crohn's disease of the pouch in patients with a preoperative

\author{
diagnosis of ulcerative colitis or \\ indeterminate colitis," explains Bo Shen of \\ the research group. \\ The development of Crohn's disease \\ in patients who have undergone IPAA \\ is associated with poor outcomes and \\ increased risk of pouch failure. The \\ researchers believe their findings will \\ directly affect daily clinical practice. They \\ suggest that patients undergoing IPAA \\ should be counseled on the risk of Crohn's \\ disease during the consent process: "in \\ patients with a family history of Crohn's \\ disease, we might tip balance in favor of \\ medical therapy or nonpouch surgery," \\ says Shen.
}

\section{Susan J. Allison}

Original article Shen, B. et al. Family history of Crohn's disease is associated with an increased risk for Crohn's disease of the pouch. Inflamm. Bowel Dis. 15, 163-170 (2009). 